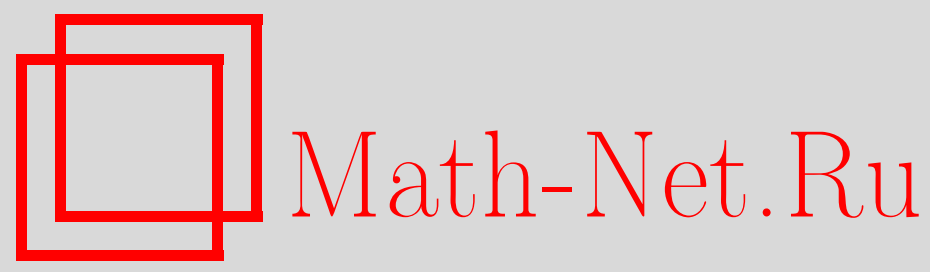

Е. Д. Нурсултанов, О применении интерполяционных методов к исследованию свойств функций многих переменных, Матем. заметки, 2004, том 75, выпуск 3, 372-383

DOI: https://doi.org/10.4213/mzm41

Использование Общероссийского математического портала Math-Net.Ru подразумевает, что вы прочитали и согласны с пользовательским соглашением http://www.mathnet.ru/rus/agreement

Параметры загрузки:

IP: 54.210 .77 .194

26 апреля 2023 г., 13:14:16 


\section{О ПРИМЕНЕНИИ ИНТЕРПОЛЯЦИОННЫХ МЕТОДОВ К ИССЛЕДОВАНИЮ СВОЙСТВ ФУНКЦИЙ МНОГИХ ПЕРЕМЕННЫХ}

\section{Е. Д. Нурсултанов}

В работе с помощью интерполяционных теорем для пространств функций многих переменных получено обобщение и уточнение в многомерном случае теоремы Хермандера о мультипликаторах преобразования $\Phi$ урье из $L_{p}$ в $L_{q}$ и неравенства Харди-Литтлвуда-Пэли для некоторого класса кратных рядов Фурье.

Библиографоия: 18 названий.

Идея “многомерной” интерполяции банаховых пространств имеет своим истоком работу А. Йошикава [1], Г. Спара [2] и получила дальнейшее развитие в статьях [3]-[10].

В данной работе мы хотим показать как применение интерполящионных теорем для пространств функций многих переменных [9], [10] позволяет получать обобщение и уточнение некоторых известных результатов.

1. Пусть $\mathbf{p}=\left(p_{1}, \ldots, p_{n}\right), \mathbf{q}=\left(q_{1}, \ldots, q_{n}\right)$ - векторы такие, что если $0<q_{j}<\infty$, то $0<p_{j}<\infty$, если же $q_{j}=\infty$, то $0<p_{j} \leqslant \infty, j=1, \ldots, n$. В дальнейшем будем считать, что векторы $\mathbf{p}$ и q удовлетворяют этим условиям.

Определим функционалы

$$
\begin{aligned}
& \Phi_{\mathbf{p q}}(\varphi)=\left(\int_{0}^{\infty} \cdots\left(\int_{0}^{\infty}\left|t_{1}^{\frac{1}{p_{1}}} \ldots t_{n}^{\frac{1}{p_{n}}} \varphi\left(t_{1}, \ldots, t_{n}\right)\right|^{q_{1}} \frac{d t_{1}}{t_{1}}\right)^{q_{2} / q_{1}} \ldots \frac{d t_{n}}{t_{n}}\right)^{1 / q_{n}} \\
& F_{\mathbf{p q}}(a)=\left(\sum_{k_{n}=1}^{\infty} k_{n}^{\frac{q_{n}}{p_{n}}-1} \ldots\left(\sum_{k_{1}=1}^{\infty} k_{1}^{\frac{q_{1}}{p_{1}}-1}\left|a_{k_{1} \ldots k_{n}}\right|^{q_{1}}\right)^{q_{2} / q_{1}} \ldots\right)^{1 / q_{n}}
\end{aligned}
$$

здесь выражения $\left(\int_{0}^{\infty}(G(t))^{q} \frac{d t}{t}\right)^{1 / q}$ и $\left(\sum_{k=1}^{\infty}\left|g_{k}\right|^{q}\right)^{1 / q}$ при $q=\infty$ понимаются как $\sup _{t>0} G(t)$ и $\sup _{k}\left|g_{k}\right|$ соответственно.

Пусть $\mathbf{m}=\left(m_{1}, \ldots, m_{n}\right) \in \mathbb{N}^{n}, f\left(x_{1}, \ldots, x_{n}\right)$ - измеримая функция, заданная в $\mathbb{R}^{\mathbf{m}}=\mathbb{R}^{m_{1}} \times \cdots \times \mathbb{R}^{m_{n}}, *=\left(j_{1}, \ldots, j_{n}\right)$ - некоторая перестановка последовательности чисел $\{1,2, \ldots, n\}$. Через $f^{*}(t)=f^{*} j_{1}, \ldots, *_{n}\left(t_{1}, \ldots, t_{n}\right)$ обозначим функцию, полученную применением невозрастающей перестановки последовательно по переменным $x_{j_{1}} \in \mathbb{R}^{m_{j_{1}}}, \ldots, x_{j_{n}} \in \mathbb{R}^{m_{j_{n}}}$, считая остальные переменные фиксированными. Данную функцию $f^{*}(t)$ будем назьвать невозрастающей перестановкой функции $f$ в $\mathbb{R}^{\mathbf{m}}$, соответствующей вектору $*=\left(j_{1}, \ldots, j_{n}\right)$. 
Пространство $L_{\mathbf{p q}}^{*}\left(\mathbb{R}^{\mathbf{m}}\right)$ определяется как множество функций, для которых

$$
\|f\|_{L_{\mathbf{p q}}^{*}\left(\mathbb{R}^{\mathbf{m}}\right)}=\Phi_{\mathbf{p q}}\left(f^{*}(\cdot)\right)<\infty
$$

Если $*=(1,2, \ldots, n)$, то пространство $L_{\mathbf{p q}}^{*}$ будем обозначать через $L_{\mathbf{p q}}$.

Пространство $L_{\mathbf{p q}}^{*}(\Omega)$, где $\Omega=\Omega_{1} \times \cdots \times \Omega_{n}, \Omega_{k} \subset \mathbb{R}^{m_{k}}$, определяется нормой

$$
\|f\|_{L_{\mathbf{p q}}^{*}(\Omega)}=\left\|f_{\Omega}\right\|_{L_{\mathbf{p q}}^{*}\left(\mathbb{R}^{\mathbf{m}}\right)}
$$

где $f_{\Omega}$ - нулевое продолжение функции $f$ с области $\Omega$ на все $\mathbb{R}^{\mathbf{m}}$.

Аналогично определяется пространство

$$
l_{\mathbf{p q}}^{*}=\left\{\left\{a_{s}\right\}_{s \in \mathbb{N}^{n}}:\|a\|_{l_{\mathbf{p q}}^{*}}=F_{\mathbf{p q}}\left(\left\{a_{k}^{*}\right\}\right)<\infty\right\}
$$

где $a_{\mathbf{k}}^{*}=a_{k_{1}, \ldots, k_{n}}^{*_{j_{1}} \cdots *_{j_{n}}}-$ невозрастающая перестановка последовательности $\left\{a_{s}\right\}_{s \in \mathbb{N} n}$, которую применили последовательно по переменным $s_{j_{1}}, \ldots, s_{j_{n}}$.

Пространства $L_{\mathbf{p q}}^{*}, l_{\mathbf{p q}}^{*}$ являются обобщением пространств Лоренца на анизотропный случай. В случае, когда $*=(1,2, \ldots, n), \mathbf{m}=(1, \ldots, 1)$, данные пространства были рассмотрены А. П. Блозинским [7] и затем изучены некоторые их свойства. В общем случае эти свойства сохраняются (см. [9]).

a) При $\mathbf{q} \leqslant \mathbf{q}_{\mathbf{1}}\left(q_{j} \leqslant q_{j}^{1}, j=1, \ldots, n\right)$ имеют место вложения

$$
L_{\mathbf{p q}}^{*}(\Omega) \hookrightarrow L_{\mathbf{p q}_{1}}^{*}(\Omega), \quad l_{\mathbf{p q}}^{*} \hookrightarrow l_{\mathbf{p q}_{1}}^{*}
$$

б) Если $p_{j_{0}}<\infty$ и область $\Omega=\Omega_{1} \times \cdots \times \Omega_{n}$ такая, что $m \Omega_{j_{0}}<\infty$, то для любого $\varepsilon>0$ имеют место вложения

$$
L_{\mathbf{p}_{\varepsilon} \mathbf{q}_{1}}^{*}(\Omega) \hookrightarrow L_{\mathbf{p q}}^{*}(\Omega), \quad l_{\mathbf{p q}_{1}}^{*} \hookrightarrow l_{\mathbf{p}_{\varepsilon} \mathbf{q}}^{*},
$$

где $\mathbf{p}=\left(p_{1}, \ldots, p_{n}\right), \mathbf{q}=\left(q_{1}, \ldots, q_{n}\right), \mathbf{p}_{\varepsilon}=\left(p_{1}, \ldots, p_{j_{0}-1}, p_{j_{0}}+\varepsilon, p_{j_{0}+1}, \ldots, p_{n}\right), \mathbf{q}_{1}=$ $\left(q_{1}, \ldots, q_{j_{0}-1}, \infty, q_{j_{0}+1}, \ldots, q_{n}\right)$.

в) (Неравенство Гёльдера). Пусть $1<\mathbf{p}, \mathbf{r}, \mathbf{q}<\infty, \mathbf{1} \leqslant \mathbf{t}, \mathbf{s}, \boldsymbol{\nu} \leqslant \infty, \mathbf{1} / \mathbf{p}+\mathbf{1} / \mathbf{r}=\mathbf{1} / \mathbf{q}$, $\mathbf{1} / \mathbf{s}+\mathbf{1} / \mathbf{t} \geqslant \mathbf{1} / \boldsymbol{\nu}\left(1 / p_{j}+1 / r_{j}=1 / q_{j}, 1 / s_{j}+1 / t_{j} \geqslant 1 / \nu_{j}, j=1, \ldots, n\right) ;$ тогда верны неравенства

$$
\|f g\|_{L_{\mathbf{q} \nu}^{*}(\Omega)} \leqslant c\|f\|_{L_{\mathbf{p s}}^{*}(\Omega)}\|g\|_{L_{\mathbf{r t}}^{*}(\Omega)}, \quad\|a b\|_{l_{\mathbf{q} \nu}^{*}} \leqslant c\|a\|_{l_{\mathbf{p s}}^{*}}\|b\|_{l_{\mathbf{r t}}^{*}}
$$

Пусть $A_{1}$ - банахово пространство, $A_{2}$ - функциональная банахова решетка. Через $A=\left(A_{1}, A_{2}\right)$ обозначим пространство $A_{1}$-значных измеримых функций таких, что $\|f(x)\|_{A_{1}} \in A_{2}$ с нормой $\|f\|=\|\| f(x)\left\|_{A_{1}}\right\|_{A_{2}}$. Пространство $\mathbf{A}=\left(A_{1}, \ldots, A_{n}\right)$ определяется индуктивно. Назовем его анизотропным пространством размерности $n$.

Пусть $\mathbf{A}_{0}=\left(A_{1}^{0}, \ldots, A_{n}^{0}\right), \mathbf{A}_{1}=\left(A_{1}^{1}, \ldots, A_{n}^{1}\right)$ - два анизотропных пространства, $E=$ $\left\{\varepsilon=\left(\varepsilon_{1}, \ldots, \varepsilon_{n}\right): \varepsilon_{j}=0\right.$, или $\left.\varepsilon_{j}=1, j=1, \ldots, n\right\}$ - вершины $n$-мерного единичного куба. Для произвольного $\varepsilon \in E$ определим пространство $\mathbf{A}_{\varepsilon}=\left(A_{1}^{\varepsilon_{1}}, \ldots, A_{n}^{\varepsilon_{n}}\right)$ с нормой

$$
\|a\|_{\mathbf{A}_{\varepsilon}}=\|\ldots\| a \|_{A_{1}^{\varepsilon_{1}} \ldots \|_{A_{n}^{\varepsilon} n}}
$$


Пару анизотропных пространств $\mathbf{A}_{0}=\left(A_{1}^{0}, \ldots, A_{n}^{0}\right), \mathbf{A}_{1}=\left(A_{1}^{1}, \ldots, A_{n}^{1}\right)$ назовем совместимой, если найдется линейное хаусдорфово пространство, содержащее в качестве подмножеств пространства $\mathbf{A}_{\varepsilon}, \varepsilon \in E$.

Пусть $*=\left(j_{1}, \ldots, j_{n}\right)$ - некоторая перестановка последовательности $(1,2, \ldots, n)$; вектору $\varepsilon=\left(\varepsilon_{1}, \ldots, \varepsilon_{n}\right) \in E$ сопоставим $\varepsilon^{*}=\left(\varepsilon_{j_{1}}, \ldots, \varepsilon_{j_{n}}\right) \in E$. Определим функционал $K^{*}$ :

$$
K^{*}\left(t, a ; \mathbf{A}_{0}, \mathbf{A}_{1}\right)=\inf \left\{\sum_{\varepsilon \in E} t^{\varepsilon}\left\|a_{\varepsilon^{*}}\right\|_{\mathbf{A}_{\varepsilon^{*}}}: a=\sum_{\varepsilon \in E} a_{\varepsilon}, \quad a_{\varepsilon} \in \mathbf{A}_{\varepsilon}\right\}
$$

где $t^{\varepsilon}=t_{1}^{\varepsilon_{1}} \ldots t_{n}^{\varepsilon_{n}}$.

Пусть $\mathbf{0}<\boldsymbol{\theta}=\left(\theta_{1}, \ldots, \theta_{n}\right)<\mathbf{1}, \mathbf{0}<\mathbf{q}=\left(q_{1}, \ldots, q_{n}\right) \leqslant \infty$. Через $\mathbf{A}_{\boldsymbol{\theta} \mathbf{q}}^{*}=\left(\mathbf{A}_{0}, \mathbf{A}_{1}\right)_{\boldsymbol{\theta} \mathbf{q}}^{*}$ обозначим линейное подмножество $\sum_{\varepsilon \in E} \mathbf{A}_{\varepsilon}$, для элементов которых верно

$$
\|a\|_{\mathbf{A}_{\mathbf{\theta}_{\mathbf{q}}}^{*}}=\Phi_{\mathbf{p q}}\left(t^{-1} K^{*}(t, a)\right)<\infty
$$

где $\mathbf{p}=\mathbf{1} /(\mathbf{1}-\boldsymbol{\theta})$.

Лемма 1. Пусть $\left(\mathbf{A}_{0}, \mathbf{A}_{1}\right)$ - совместимая пара анизотропных пространств, $E=\left\{\varepsilon=\left(\varepsilon_{1}, \ldots, \varepsilon_{n}\right): \varepsilon_{j}=0\right.$ или $\left.\varepsilon_{j}=1, \quad i=1, \ldots, n\right\}$. Пусть $*=\left(j_{1}, \ldots, j_{n}\right), *_{1}=$ $\left(r_{1}, \ldots, r_{n}\right)$ - некоторые перестановки последовательности $(1, \ldots, n) ;$ выражение * о $*_{1}=\left(r_{j_{1}}, \ldots, r_{j_{n}}\right)$ определяет перестановку последовательности $\left(r_{1}, \ldots, r_{n}\right)$, соответствующую вектору $*=\left(j_{1}, \ldots, j_{n}\right)$. Если $T$ - линейный оператор такой, что $T: \mathbf{A}_{\varepsilon^{*}} \rightarrow \mathbf{B}_{\varepsilon}$, с нормой $M_{\varepsilon}$ для любого $\varepsilon \in E$, mо

$$
T: \mathbf{A}_{\boldsymbol{\theta} \mathbf{q}}^{* *_{1}} \rightarrow \mathbf{B}_{\boldsymbol{\theta} \mathbf{q}}^{*_{1}}, \quad \mathbf{0}<\boldsymbol{\theta}<\mathbf{1}, \quad \mathbf{0}<\mathbf{q} \leqslant \infty,
$$

с нормой $\|T\| \leqslant \max _{\varepsilon \in E} M_{\varepsilon}$.

Следующее утверждение является следствием теорем 1,2 из [9].

Teоpema 1. Пусть $\mathbf{1} \leqslant \mathbf{p}_{\mathbf{i}}=\left(p_{1}^{i}, \ldots, p_{n}^{i}\right), \boldsymbol{\sigma}_{\mathbf{i}}=\left(\sigma_{1}^{i}, \ldots, \sigma_{n}^{i}\right) \leqslant \infty, i=0,1, \quad \mathbf{p}_{\mathbf{0}} \neq$ $\mathbf{p}_{\mathbf{1}}, \quad \mathbf{0}<\mathbf{q}=\left(q_{1}, \ldots, q_{n}\right) \leqslant \infty, \quad \mathbf{0}<\boldsymbol{\theta}=\left(\theta_{0}, \ldots, \theta_{n}\right)<\mathbf{1}, \quad \mathbf{1} / \mathbf{p}=(\mathbf{1}-\boldsymbol{\theta}) / \mathbf{p}_{\mathbf{0}}+\boldsymbol{\theta} / \mathbf{p}_{\mathbf{1}}$, * = $\left(j_{1}, \ldots, j_{n}\right)$ - некоторая перестановка последовательности $\{1,2, \ldots, n\}$, $\mathbf{A}_{\mathbf{p}_{0} \sigma_{0}}^{*}=\left(L_{p_{j_{1}}^{0} \sigma_{j_{1}}^{0}}, \ldots, L_{p_{j_{n}}^{0} \sigma_{j_{n}}^{0}}\right), \quad \mathbf{A}_{\mathbf{p}_{1} \sigma_{1}}^{*}=\left(L_{p_{j_{1} \sigma_{j_{1}}^{1}}^{1}}, \ldots, L_{p_{j_{n}}^{1} \sigma_{j_{n}}^{1}}\right), \quad \mathbf{B}_{\mathbf{p}_{0} \sigma_{0}}^{*}=$

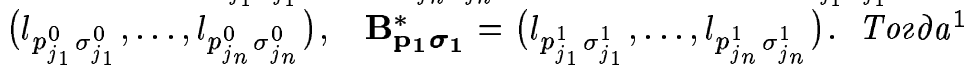

$$
\begin{array}{ll}
L_{\mathbf{p q}}^{*}=\left(\mathbf{A}_{\mathbf{p}_{0} \sigma_{0}}^{*}, \mathbf{A}_{\mathbf{p}_{1} \sigma_{1}}^{*}\right)_{\theta \mathbf{q}}^{*}, & l_{\mathbf{p q}}^{*}=\left(\mathbf{B}_{\mathbf{p}_{0} \sigma_{0}}^{*}, \mathbf{B}_{\mathbf{p}_{1} \sigma_{1}}^{*}\right)_{\theta \mathbf{q}}^{*}, \\
L_{\mathbf{p q}}^{*}=\left(L_{\mathbf{p}_{0} \infty}^{*}, L_{\mathbf{p}_{1 \infty}}^{*}\right)_{\theta \mathbf{q}}^{*}, & l_{\mathbf{p q}}^{*}=\left(l_{\mathbf{p}_{0} \infty}^{*}, l_{\mathbf{p}_{1} \infty}^{*}\right)_{\theta \mathbf{q}}^{*}
\end{array}
$$

\footnotetext{
${ }^{1}$ Здесь $L_{p_{j_{s}}^{i}} \sigma_{j_{s}}^{i}, l_{j_{s}}^{i} \sigma_{j_{s}}^{i}$ - обычные (со скалярными параметрами) пространства Лоренца.
} 
ЗАмЕчАниЕ. Как видно из теоремы 1, порядок расстановки (интегрирование) пространств в $\mathbf{A}_{\mathbf{p}_{\mathbf{i}} \boldsymbol{\sigma}_{\mathbf{i}}}^{*}=\left(L_{p_{j_{1}}^{i} \sigma_{j_{1}}^{i}}, \ldots, L_{p_{j_{n}}^{i} \sigma_{j_{n}}^{i}}\right), i=0,1$, предопределяет порядок невозрастающей перестановки в $L_{\mathbf{p q}}^{*}=\left(\mathbf{A}_{\mathbf{p}_{0} \boldsymbol{\sigma}_{0}}^{*}, \mathbf{A}_{\mathbf{p}_{\mathbf{1}} \boldsymbol{\sigma}_{\mathbf{1}}}^{*}\right)_{\theta \mathbf{q}}^{*}$. Например, интерполировние одним и тем же методом пары пространств $\left(L_{p_{0}}\left(\mathbb{R}_{x}\right), L_{p_{0}}\left(\mathbb{R}_{y}\right)\right),\left(L_{p_{1}}\left(\mathbb{R}_{x}\right), L_{p_{1}}\left(\mathbb{R}_{y}\right)\right)$ и $\left(L_{p_{0}}\left(\mathbb{R}_{y}\right), L_{p_{0}}\left(\mathbb{R}_{x}\right)\right),\left(L_{p_{1}}\left(\mathbb{R}_{y}\right), L_{p_{1}}\left(\mathbb{R}_{x}\right)\right)$ дает два разных результата, хотя как линейные нормированные пространства эти пары совпадают:

$$
L_{p_{i}}\left(\mathbb{R}^{2}\right)=\left(L_{p_{i}}\left(\mathbb{R}_{x}\right), L_{p_{i}}\left(\mathbb{R}_{y}\right)\right)=\left(L_{p_{i}}\left(\mathbb{R}_{y}\right), L_{p_{i}}\left(\mathbb{R}_{x}\right)\right), \quad i=0,1 .
$$

Порядок же интегрирования в $L_{\mathbf{p q}}^{*}=\left(\mathbf{A}_{\mathbf{p}_{0} \boldsymbol{\sigma}_{0}}^{*}, \mathbf{A}_{\mathbf{p}_{\mathbf{1}} \boldsymbol{\sigma}_{\mathbf{1}}}\right)_{\theta \mathbf{q}}^{*}$ определяется самим интерполящионным методом.

2. Пусть $F, F^{-1}$ - прямое и обратное преобразования Фурье в $\mathbb{R}^{n}$. Функцию $\varphi$ назовем мультипликатором преобразования Фурье из функционального пространства Лоренца $L_{\mathbf{p} \boldsymbol{\tau}}\left(\mathbb{R}^{n}\right)$ в пространство Лоренца $L_{\mathbf{q s}}\left(\mathbb{R}^{n}\right)$, если оператор $T_{\varphi}(f)=F^{-1} \varphi F f$ является ограниченным из пространства $L_{\mathbf{p} \boldsymbol{\tau}}\left(\mathbb{R}^{n}\right)$ в $L_{\mathbf{q s}}\left(\mathbb{R}^{n}\right)$. Совокупность всех мультипликаторов из $L_{\mathbf{p} \boldsymbol{\tau}}$ в $L_{\mathbf{q s}}$ обозначим через $M\left(L_{\mathbf{p} \boldsymbol{\tau}} \rightarrow L_{\mathbf{q s}}\right)$. Данное множество является линейным нормированным пространством с нормой

$$
\|\varphi\|_{M\left(L_{\mathbf{p} \tau} \rightarrow L_{\mathbf{q s}}\right)}=\sup _{f \neq 0} \frac{\left\|T_{\varphi}(f)\right\|_{L_{\mathbf{q s}}}}{\|f\|_{L_{\mathbf{p} \tau}}} .
$$

Теорема 2. Пусть $F-$ преобразование Фурье, $\mathbf{2}<\mathbf{p}=\left(p_{1}, \ldots, p_{n}\right)<\infty, \mathbf{p}^{\prime}=$ $\mathbf{p} /(\mathbf{p}-\mathbf{1}), \quad \mathbf{0}<\mathbf{q}=\left(q_{1}, \ldots, q_{n}\right) \leqslant \infty, *=\left(j_{1}, \ldots, j_{n}\right)$ - некоторая перестановка последовательности $(1,2, \ldots, n), *^{\prime}=\left(j_{n}, \ldots, j_{1}\right)$. Тогда

$$
\|F f\|_{L_{\mathbf{p q}}^{*}\left(\mathbb{R}^{n}\right)} \leqslant c\|f\|_{L_{\mathbf{p}^{\prime} \mathbf{q}}^{*^{\prime}}\left(\mathbb{R}^{n}\right)} .
$$

ДокАЗАТЕЛЬСтво проведем в случае, когда $*=(1,2, \ldots, n)$. Пусть $\mathbf{p}_{0}=\left(p_{1}^{0}, \ldots\right.$, $\left.p_{n}^{0}\right)=(2, \ldots, 2), \mathbf{p}_{1}=\left(p_{1}^{1}, \ldots, p_{n}^{1}\right)=(\infty, \ldots, \infty), E=\left\{\varepsilon=\left(\varepsilon_{1}, \ldots, \varepsilon_{n}\right): \varepsilon_{i}=0\right.$ или $\left.\varepsilon_{i}=1, i=1, \ldots, n\right\}$. Введем обозначение $\mathbf{p}_{\varepsilon}=\left(p_{\varepsilon_{1}}, \ldots, p_{\varepsilon_{n}}\right)=\left(p_{1}^{\varepsilon_{1}}, \ldots, p_{n}^{\varepsilon_{n}}\right)$. Покажем, что для любого $\varepsilon \in E$ верно

$$
\|F f\|_{L_{\mathbf{p}_{\varepsilon}}} \leqslant\|\ldots\|\|f\|_{L_{p_{\varepsilon_{n}}^{\prime}}}\left\|{ }_{L_{p_{\varepsilon_{n-1}}^{\prime}}} \ldots\right\|_{L_{p_{\varepsilon_{1}}^{\prime}}}
$$

здесь $1 / p_{\varepsilon_{j}}^{\prime}+1 / p_{\varepsilon_{j}}=1, j=1, \ldots, n$. Для $n=1$ доказательство следует из равенства Парсеваля и определения преобразования Фурье. Предположим, что неравенство (1) справедливо для $n$. Покажем, что оно верно для значения $n+1$. Пусть $\varepsilon_{n+1}=0$ т.е. $p_{\varepsilon_{n+1}}=2, \mathbf{p}_{\varepsilon}=\left(p_{\varepsilon_{1}}, \ldots, p_{\varepsilon_{n}}\right), x=\left(x_{1}, \ldots, x_{n}\right), y=\left(y_{1}, \ldots, y_{n}\right)$. Используя предположение индукции, имеем

$$
\begin{aligned}
& \|F f\|_{L_{\left(\mathbf{p} \varepsilon, p_{\varepsilon_{n+1}}\right)}\left(\mathbb{R}^{n+1}\right)}^{2}=\int_{-\infty}^{\infty}\|F f\|_{L_{\mathbf{p}_{\varepsilon}}\left(\mathbb{R}^{n}\right)}^{2} d y_{n+1} \\
& \quad=\int_{-\infty}^{\infty}\left\|\int_{\mathbb{R}^{n}}\left(\int_{\infty}^{\infty} f\left(x, x_{n+1}\right) e^{-i y_{n+1} x_{n+1}} d x_{n+1}\right) e^{-i y x} d x\right\|_{L_{\mathbf{p}_{\varepsilon}}\left(\mathbb{R}^{n}\right)}^{2} d y_{n+1} \\
& \quad \leqslant \int_{-\infty}^{\infty}\left(\|\ldots\|\left\|\int_{\infty}^{\infty} f\left(\cdot, x_{n+1}\right) e^{-i y_{n+1} x_{n+1}} d x_{n+1}\right\|_{L_{p_{\varepsilon_{n}}^{\prime}}} \|_{\left.L_{p_{\varepsilon_{n-1}}^{\prime}} \ldots \|_{L_{\varepsilon_{\varepsilon_{1}}}}\right)^{2 / p_{\varepsilon_{1}}^{\prime}} d y_{n+1} .}\right.
\end{aligned}
$$


Так как $p_{\varepsilon_{j}}^{\prime} \leqslant 2, j=1, \ldots, n$, из неравенства Минковского получим $(1)$. Если $\varepsilon_{n+1}=1$, т.е. $p_{\varepsilon_{n+1}}=\infty$, то, сначала применяя неравенство Минковского, а затем предположение индукции, получим неравенство (1).

Таким образом, используя интерполяционные свойства пространств $L_{\mathbf{p}}$ (теорема 1 , лемма 1), получим требуемое утверждение.

Tеорема 3. Пусть $*=(n, n-1, \ldots, 1), \quad \mathbf{1}<\mathbf{p}<\mathbf{2}<\mathbf{q}<\infty, \quad \mathbf{0}<\mathbf{s}, \mathbf{t} \leqslant \infty$,

$$
\frac{1}{\mathbf{r}}=\frac{1}{\mathbf{p}}-\frac{1}{\mathrm{q}}, \quad \frac{1}{\mathbf{h}}=\left(\frac{1}{\mathrm{~s}}-\frac{1}{\mathbf{t}}\right)_{+} ;
$$

здесь $(x)_{+}=\max (0, x)$. Тогда

$$
L_{\mathbf{r h}}^{*} \hookrightarrow M\left(L_{\mathbf{p t}} \rightarrow L_{\mathbf{q s}}\right) .
$$

ДокАЗАТЕЛьСТво. Воспользуемся теоремой 2, неравенством Гёльдера, а также свойствами пространств $L_{\mathbf{p t}}^{*}$; получим

$$
\left\|F^{-1} \varphi F f\right\|_{L_{\mathbf{q s}}} \leqslant c_{1}\|\varphi F f\|_{L_{\mathbf{q}^{\prime} \mathbf{s}}^{*}} \leqslant c_{1}\|\varphi\|_{L_{\mathbf{r h}}^{*}}\|F f\|_{L_{\mathbf{p}^{\prime} \mathbf{t}}^{*}} \leqslant c_{2}\|\varphi\|_{L_{\mathbf{r h}}^{*}}\|f\|_{L_{\mathbf{p t}}}
$$

что и требовалось доказать.

В изотропном случае, при $\mathbf{p}=(p, \ldots, p)=\mathbf{t}, \mathbf{q}=(q, \ldots, q)=\mathbf{s}, 1<p<2<q \leqslant \infty$, $1 / r=1 / p-1 / q$ из теоремы 3 следует

$$
\|\varphi\|_{M\left(L_{p} \rightarrow L_{q}\right)} \leqslant \sup _{t_{j}>0} t_{1}^{1 / r} \ldots t_{n}^{1 / r} \varphi^{*_{1} \cdots *_{n}}\left(t_{1}, \ldots, t_{n}\right)=\|\varphi\|_{L_{(r, \ldots, r)(\infty, \ldots, \infty)}\left(\mathbb{R}^{n}\right)} .
$$

Данное утверждение более точное, чем утверждение теоремы Хермандера [11]: при $1<$ $p<2 \leqslant q \leqslant \infty, 1 / r=1 / p-1 / q$

$$
\|\varphi\|_{M\left(L_{p} \rightarrow L_{q}\right)} \leqslant c\|\varphi\|_{L_{r \infty}\left(\mathbb{R}^{n}\right)} .
$$

Действительно, для простоты рассуждений положим $n=2$. Тогда

$$
\begin{aligned}
\|\varphi\|_{L_{(r, r)(\infty, \infty)}} & =\sup _{t_{1}>0, t_{2}>0}\left(t_{1} t_{2}\right)^{1 / r} \varphi^{*_{1} *_{2}}\left(t_{1}, t_{2}\right) \\
& \leqslant \sup _{t_{1}>0, t_{2}>0} \frac{1}{t_{1}^{1-1 / r}} \frac{1}{t_{2}{ }^{1-1 / r}} \int_{0}^{t_{2}} \int_{0}^{t_{1}} \varphi^{*_{1} *_{2}}\left(s_{1}, s_{2}\right) d s_{1} d s_{2} \\
& \leqslant \sup _{t_{1}>0, t_{2}>0} \sup _{\left|e_{2}\right|=t_{2}} \frac{1}{\left|e_{2}\right|} \int_{e_{2}\left|e_{1}\right|=t_{1}} \sup _{\left|e_{1}\right|} \int_{e_{1}}\left|\varphi\left(x_{1}, x_{2}\right)\right| d x_{1} d x_{2} .
\end{aligned}
$$

Из определения точной верхней грани следует, что для произвольньх фиксированных $t_{1}>0, t_{2}>0$ найдется семейства измеримых множеств $e_{2}\left(t_{2}\right),\left\{e_{1}\left(t_{1}, x_{2}\right)\right\}_{x_{2} \in e_{2}\left(t_{2}\right)}$, что $\left|e_{2}\left(t_{2}\right)\right|=t_{2},\left|e_{1}\left(t_{1}, x_{2}\right)\right|=t_{1}$ и

$$
\begin{aligned}
\|\varphi\|_{L_{(r, r)(\infty, \infty)} \leqslant} \leqslant & 4 \sup _{t_{1}>0, t_{2}>0} \frac{1}{\left|e_{2}\left(t_{2}\right)\right|^{1-1 / r}} \int_{e_{2}\left(t_{2}\right)} \\
& \times \frac{1}{\left|e_{1}\left(t_{1}, x_{2}\right)\right|^{1-1 / r}} \int_{e_{1}\left(t_{1}, x_{2}\right)}\left|\varphi\left(x_{1}, x_{2}\right)\right| d x_{1} d x_{2} \\
= & 4 \sup _{t_{1}>0, t_{2}>0} \frac{1}{\left(t_{1} t_{2}\right)^{1-1 / r}} \int_{e_{2}\left(t_{2}\right)} \int_{e_{1}\left(t_{1}, x_{2}\right)}\left|\varphi\left(x_{1}, x_{2}\right)\right| d x_{1} d x_{2} \\
\leqslant & 4 \sup _{|e|>0} \frac{1}{|e|^{1-1 / r}} \int_{e}|\varphi(x)| d x \sim\|\varphi\|_{L_{r \infty} \cdot}
\end{aligned}
$$

Следуюший пример показывает, что пространства $L_{r \infty}\left(\mathbb{R}^{2}\right)$ и $L_{(r, r)(\infty, \infty)}\left(\mathbb{R}^{2}\right)$ различны. 
ПРИмеР 1. Пусть $1<r<\infty$. Рассмотрим функцию

$$
\varphi\left(x_{1}, x_{2}\right)=\left(\left|x_{1}\right|\left|x_{2}\right|\right)^{-1 / r}
$$

Очевидно, что $\sup _{t_{1}>0, t_{2}>0} t_{1}^{1 / r} t_{2}^{1 / r} \varphi^{*_{1} *_{2}}\left(t_{1}, t_{2}\right)<\infty$. Покажем, что $\|\varphi\|_{L_{r \infty}}=$ $\sup _{t>0} t^{1 / r} \varphi^{*}(t)=\infty$. Действительно, для $\varepsilon>0$ определим множество $e_{\varepsilon}=\left\{\left(x_{1}, x_{2}\right)\right.$ : $\left.\varepsilon \leqslant x_{1} \leqslant 1,0 \leqslant x_{2} \leqslant \varepsilon / x_{1}\right\}$. Тогда

$$
\frac{1}{\left|e_{\varepsilon}\right|^{1-1 / r}} \int_{e_{\varepsilon}} \varphi\left(x_{1}, x_{2}\right) d x_{1} d x_{2} \sim|\ln \varepsilon|^{1 / r} \rightarrow \infty, \quad \varepsilon \rightarrow 0
$$

и, следовательно,

$$
\|g\|_{L_{r \infty}} \sim \sup _{|e|>0} \frac{1}{|e|^{1-1 / r}}\left|\int_{e} \varphi(x) d x\right|=\infty
$$

Функцию $f$ назовем обобщенно-монотонной (невозрастающей) в $\mathbb{R}^{n}$, если для любого $x \in \mathbb{R}^{n}$ и $x_{j} \neq 0, j=1, \ldots, n$, имеет место

$$
|f(x)| \leqslant \frac{B}{\left|x_{1}\right| \ldots\left|x_{n}\right|}\left|\int_{[0, x]} f(y) d y\right|
$$

здесь $[0, x]=\left\{y \in \mathbb{R}^{n}: 0 \leqslant y_{j} \operatorname{sign} x_{j} \leqslant\left|x^{j}\right|, j=1, \ldots, n\right\}$.

СлЕДСТВИЕ 1. Пусть $1<p<2<q<\infty, 1 \leqslant \tau \leqslant s \leqslant \infty, \varphi-$ знакоопределенная, обобщ,нно-монотонная функция. Тогда для того, чтобы $\varphi \in M\left(L_{p \tau} \rightarrow L_{q s}\right)$, необходимо и достаточно, чтобы

$$
\sup _{Q \in E_{0}} \frac{1}{|Q|^{1 / p^{\prime}+1 / q}} \int_{Q}|\varphi(x)| d x<\infty
$$

әде $E_{0}$ - множество всех параллелепипедов с гранями, параллельными декартовым плоскостям.

ЗАмЕчАниЕ. Данное утверждение было сформулировано в заметке автора [12] (следствие 1). Приведенное там доказательство является верным лишш для одномерного случая $(n=1)$.

ДокАЗАТЕЛЬСТВо. Пусть $1 / r=1 / p-1 / q$. Из теоремы 1 работы [12] следует, что для знакоопределенной функции $\varphi$ из $M\left(L_{p \tau} \rightarrow L_{q s}\right)$ верна оценка

$$
\sup _{Q \in E_{0}} \frac{1}{|Q|^{1-1 / r}}\left|\int_{Q} \varphi(x) d x\right| \leqslant\|\varphi\|_{M\left(L_{p \tau} \rightarrow L_{q s}\right)} .
$$

Зафиксируем параметр $r \in(1, \infty)$ и функцию $f \in L_{(r \ldots r)(\infty \ldots \infty)}\left(\mathbb{R}^{n}\right)$. Тогда для пар $\left(p_{0}, q_{0}\right),\left(p_{1}, q_{1}\right)$ таких, что $1 / p_{0}-1 / q_{0}=1 / r$, из соотношения $(2)$ имеем

$$
\left\|T_{\varphi} f\right\|_{L_{q_{i}}\left(\mathbb{R}^{n}\right)} \leqslant c\|\varphi\|_{L_{(r \ldots r)(\infty \ldots \infty)}\left(\mathbb{R}^{n}\right)}\|f\|_{L_{p_{i}}\left(\mathbb{R}^{n}\right)}, \quad i=0,1 .
$$


Применяя вещественный метод интерполяции, получим: при $\tau \leqslant s, 1 / p-1 / q=1 / r$

$$
\left\|T_{\varphi} f\right\|_{L_{q s}\left(\mathbb{R}^{n}\right)} \leqslant c\|\varphi\|_{L_{(r \ldots r)(\infty \ldots \infty)}\left(\mathbb{R}^{n}\right)}\|f\|_{L_{p \tau}\left(\mathbb{R}^{n}\right)},
$$

т.e.

$$
\begin{aligned}
& \|\varphi\|_{M\left(L_{p \tau} \rightarrow L_{q s}\right)} \leqslant c \sup _{t_{1}>0, \ldots, t_{n}>0} t_{1}^{1 / r} \ldots t_{n}^{1 / r} \varphi^{*_{1} \cdots * n}\left(t_{1} \ldots t_{n}\right) \\
& \quad \leqslant c \sup _{\left|e_{n}\right|>0} \frac{1}{\left|e_{n}\right|^{1-1 / r}}\left(\int_{e_{n}} \ldots \sup _{\left|e_{1}\right|>0}\left(\frac{1}{\left|e_{1}\right|^{1-1 / r}} \int_{e_{1}} \varphi\left(x_{1}, \ldots, x_{n}\right) d x_{1}\right) \ldots d x_{n}\right) .
\end{aligned}
$$

Из условия обобщено-монотонности функции $\varphi$ следует

$$
\begin{aligned}
\sup _{\left|e_{n}\right|>0} & \frac{1}{\left|e_{n}\right|^{1-1 / r}}\left(\int_{e_{n}} \ldots \sup _{\left|e_{1}\right|>0}\left(\frac{1}{\left|e_{1}\right|^{1-1 / r}} \int_{e_{1}} \varphi\left(x_{1}, \ldots, x_{n}\right) d x_{1}\right) \ldots d x_{n}\right) \\
\leqslant & B \sup _{\left|e_{n}\right|>0} \frac{1}{\left|e_{n}\right|^{1-1 / r}} \\
& \times\left(\int_{e_{n}} \ldots \sup _{\left|e_{1}\right|>0}\left(\frac{1}{\left|e_{1}\right|^{1-1 / r}} \int_{e_{1}} \frac{1}{\left|x_{1}\right| \ldots\left|x_{n}\right|} \int_{[0, x]} \varphi(y) d y d x_{1}\right) \ldots d x_{n}\right) \\
\leqslant & B \sup _{Q \in E_{0}} \frac{1}{|Q|^{1 / p^{\prime}+1 / q}} \int_{Q} \varphi(x) d x \\
& \times \sup _{\left|e_{n}\right|>0} \frac{1}{\left|e_{n}\right|^{1-1 / r}}\left(\int_{e_{n}} \ldots \sup _{\left|e_{1}\right|>0}\left(\frac{1}{\left|e_{1}\right|^{1-1 / r}} \int_{e_{1}} \frac{1}{\left(\left|x_{1}\right| \ldots\left|x_{n}\right|\right)^{\frac{1}{r}}} d x_{1}\right) \ldots d x_{n}\right) \\
= & B c \sup _{Q \in E_{0}} \frac{1}{|Q|^{1 / p^{\prime}+1 / q}}\left|\int_{Q} \varphi(x) d x\right| .
\end{aligned}
$$

Следствие доказано.

3. Неравенство Харди-Литтлвуда-Пэли для рядов $f=\sum_{k=1}^{\infty} a_{k} \varphi_{k}(x)$ по ортонормированной в $L_{2}[0,1]^{n}$ системе $\Phi=\left\{\varphi_{k}\right\},\left|\varphi_{k}(x)\right| \leqslant c$, имеет вид:

$$
\begin{array}{ll}
\|f\|_{L_{p}[0,1]^{n}}^{p} \leqslant c \sum_{i=1}^{\infty} i^{p-2}\left(a_{i}^{*}\right)^{p} & \text { при } 2<p<\infty, \\
\sum_{i=1}^{\infty} i^{p-2}\left(a_{i}^{*}\right)^{p} \leqslant c\|f\|_{L_{p}[0,1]^{n}}^{p} \quad \text { при } 1<p<2,
\end{array}
$$

где $\left\{a_{i}^{*}\right\}_{i=1}^{\infty}-$ невозрастающая перестановка последовательности $\left\{a_{k}\right\}_{k \in \mathbb{N} n}$ коэффициентов Фурье функции $f$ по системе $\Phi$.

В одномерном случае неравенства (3) и (4) самые точные среди различных вариантов подобных неравенств (Харди-Литтлвуда, Хаусдорфа-Юнга [13], Пита [14] и др. [15], [16]). Для кратньх рядов Фурье ситуация иная; например, для тригонометрических систем имеются еще независимые неравенства [15], [16]:

$$
\|f\|_{L_{p}[0,1]^{n}}^{p} \leqslant \sum_{k_{1}=0}^{\infty} \cdots \sum_{k_{n}=0}^{\infty} 2^{\left(\frac{p}{2}-1\right)\left(k_{1}+\cdots+k_{n}\right)}\left(\sum_{m_{1}=2^{k_{1}}}^{2^{k_{1}+1}-1} \cdots \sum_{m_{n}=2^{k_{n}}}^{2^{k_{n}+1}-1}\left|a_{m_{1} \ldots m_{n}}\right|^{2}\right)^{\frac{p}{2}}
$$


при $2<p<\infty, f \sim \sum_{m \in \mathbb{N}^{n}} a_{m} \exp \left(2 \pi i \sum_{r=1}^{n} m_{r} x_{r}\right)$,

$$
\sum_{k_{1}=0}^{\infty} \cdots \sum_{k_{n}=0}^{\infty} 2^{\left(\frac{p}{2}-1\right)\left(k_{1}+\cdots+k_{n}\right)}\left(\sum_{m_{1}=2^{k_{1}}}^{2^{k_{1}+1}-1} \cdots \sum_{m_{n}=2^{k_{n}}}^{2^{k_{n}+1}-1}\left|a_{m_{1} \ldots m_{n}}\right|^{2}\right)^{\frac{p}{2}} \leqslant c\|f\|_{L_{p}[0,1]^{n}}^{p},
$$

при $1<p<2$. В этом пункте приводятся неравенства из которых следуют (3)-(6).

Пусть $\mathbf{m}=\left(m_{1}, \ldots, m_{n}\right), m_{j} \in \mathbb{N}, \Phi_{j}=\left\{\varphi_{k}^{j}\left(x_{j}\right)\right\}_{k \in \mathbb{N}}$ - ортонормированные системы функций $(\mathrm{OHC} \Phi)$ в $L_{2}[0,1]^{m_{j}}$ такие, что

$$
\sup _{x_{j} \in[0,1]^{m}, k \in \mathbb{N}}\left|\varphi_{k}^{j}\left(x_{j}\right)\right| \leqslant c, \quad j=1,2, \ldots, n .
$$

Система $\Psi=\left\{\psi_{k}(x)\right\}_{k \in \mathbb{N}^{n}}, x \in[0,1]^{\mathbf{m}}=[0,1]^{m_{1}} \times \cdots \times[0,1]^{m_{n}}$,

$$
\psi_{k}(x)=\varphi_{k}^{1}\left(x_{1}\right) \ldots \varphi_{k}^{n}\left(x_{n}\right)
$$

является ОНСФ в $L_{2}[0,1]^{\mathbf{m}}$ и ограниченная в совокупности.

Теорема 4. Пусть $\Psi-\operatorname{OHC} \Phi(7), f \sim \sum_{k \in \mathbb{N}^{n}} a_{k} \psi_{k}(x), \quad *=\left(j_{1}, \ldots, j_{n}\right)-$ некоторая перестановка последовательности $(1,2, \ldots, n), *^{\prime}=\left(j_{n}, j_{n-1}, \ldots, j_{1}\right)$. Тогда

$$
\|f\|_{L_{\mathbf{p q}}^{*}[0,1]^{\mathbf{m}}} \leqslant c\|a\|_{l_{\mathbf{p}^{\prime} \mathbf{q}}^{*^{\prime}}},
$$

при $\mathbf{2}<\mathbf{p}=\left(p_{1}, \ldots, p_{n}\right)<\infty, \quad \mathbf{0}<\mathbf{q}=\left(q_{1}, \ldots, q_{n}\right) \leqslant \infty, \mathbf{p}^{\prime}=\mathbf{p} /(\mathbf{p}-\mathbf{1})$,

$$
\|a\|_{\substack{l^{\mathbf{p}^{\prime} \mathbf{q}}\\}} \leqslant c\|f\|_{L_{\mathbf{p q}}^{*}[0,1]^{\mathbf{m}}}
$$

$n$ pu $\mathbf{1}<\mathbf{p}=\left(p_{1}, \ldots, p_{n}\right)<\mathbf{2}, \quad \mathbf{0}<\mathbf{q}=\left(q_{1}, \ldots, q_{n}\right) \leqslant \infty, \mathbf{p}^{\prime}=\mathbf{p} /(\mathbf{p}-\mathbf{1})$.

Данные неравенства при $*=(1,2, \ldots, n)$ и $\mathbf{m}=(1,1, \ldots, 1)$ были доказаны в работе [10]. Доказательство теоремы 4 проводится с применением интерполяционной теоремы 1 аналогично доказательству теоремы 2 (см. также теорему 7 из [10]).

Отметим, что порядок невозрастающих перестановок в теоремах 2 и 4 имеет существенное значение. Так, неравенство

$$
\|a\|_{l_{\mathbf{p}^{\prime} \mathbf{q}}^{*}} \leqslant c\|f\|_{L_{\mathbf{p q}}^{*}[0,1]^{\mathbf{m}}}
$$

неверно (здесь в левой части неравенства вместо положенного $*^{\prime}$ стоит $*$ ). Действительно, пусть $n=2, *=(1,2), 1<p_{1}<p_{2}<2, q_{2}<\infty, \varphi(x) \sim \sum_{k=1}^{\infty} \frac{1}{k^{1 / p_{2}^{\prime}}} \exp (2 \pi i k x)$. Рассмотрим функцию $f(x, y)=\varphi(x+y)$; ее коэффищиенты Фурье

$$
a_{k_{1} k_{2}}= \begin{cases}k^{-1 / p_{2}^{\prime}}, & k_{1}=k_{2}=k>0, \\ 0 & \text { в остальных случаях, }\end{cases}
$$

$k_{1}, k_{2} \in \mathbb{N}$. Тогда общий член невозрастающей перестановки $\left\{a_{k_{1} k_{2}}^{*_{1} *_{2}}\right\}$ имеет вид

$$
a_{k_{1} k_{2}}^{*_{1} *_{2}}= \begin{cases}k_{2}^{-1 / p_{2}^{\prime}}, & k_{1}=1 \\ 0, & k_{1}>1\end{cases}
$$


Поэтому $\|a\|_{l_{\mathbf{p}^{\prime} \mathbf{q}}^{*}}=\left\|\left\{1 / k^{1 / p_{2}^{\prime}}\right\}\right\|_{l_{p_{2}^{\prime} q_{2}}}=\infty$. С другой стороны, $f^{*_{1}}(t, y)=\varphi^{*}(t)$ и $\|f\|_{L_{\mathbf{p q}}^{*}}=\|\varphi\|_{L_{p_{1} q_{1}}} \sim\left\|\left\{1 / k^{1 / p_{2}^{\prime}}\right\}\right\|_{l_{p_{1}^{\prime} q_{1}}}<\infty$; следовательно, неравенство (8) не имеет места.

Если $\mathbf{m}=(1, \ldots, 1), \mathbf{p}=(p, \ldots, p)=\mathbf{q}$, то из теоремы 4 следуют неравенства:

$$
\begin{array}{cl}
\|f\|_{L_{p}[0,1]^{n}}^{p} \leqslant c \sum_{k_{1}=1}^{\infty} \ldots \sum_{k_{n}=1}^{\infty} k_{1}^{p-2} \ldots k_{n}^{p-2}\left(a_{k_{1} k_{2} \ldots k_{n}}^{* k_{j_{1}} j_{2} \cdots *_{n}}\right)^{p} & \text { при } 2<p<\infty, \\
\sum_{k_{1}=1}^{\infty} \ldots \sum_{k_{n}=1}^{\infty} k_{1}^{p-2} \ldots k_{n}^{p-2}\left(a_{k_{1} k_{2} \ldots k_{n}}^{* j_{j_{1}} j_{j} \cdots * j_{n}}\right)^{p} \leqslant c\|f\|_{L_{p}[0,1]^{n}}^{p} & \text { при } 1<p<2 .
\end{array}
$$

Покажем, что данные неравенства в случае системы вида (7) более точные, чем (3) и (4). Пусть $A_{r}=\left\{\mathbf{m} \in \mathbb{N}^{n}: m_{1} \cdot m_{2} \ldots m_{n}=r\right\}, r \in \mathbb{N}$,

$$
\sum_{k_{1}=1}^{\infty} \ldots \sum_{k_{n}=1}^{\infty} k_{1}^{p-2} \ldots k_{n}^{p-2}\left(a_{k_{1} \ldots k_{n}}^{*_{1} \cdots *_{n}}\right)^{p}=\sum_{r=1}^{\infty} r^{p-2} \sum_{\mathbf{m} \in A_{r}}\left(a_{m_{1} \ldots m_{n}}^{*_{1} \cdots *_{n}}\right)^{p}
$$

С другой стороны,

$$
\sum_{r=1}^{\infty} r^{p-2}\left(a_{r}^{*}\right)^{p}=\sum_{r=1}^{\infty} \sum_{\mathbf{m} \in A_{r}} \beta_{\mathbf{m}}^{p-2}\left(a_{m_{1} \ldots m_{n}}^{*_{1} \cdots m_{n}}\right)^{p}
$$

здесь последовательность $\left\{\beta_{\mathbf{m}}^{p-2}\right\}_{\mathbf{m} \in \mathbb{N} n}$ есть соответствующая перестановка последовательности $\left\{r^{p-2}\right\} r \in \mathbb{N}$.

Учитывая, что $a_{k_{1} \ldots k_{n}}^{*_{1} \cdots *_{n}} \geqslant a_{m_{1} \ldots m_{n}}^{*_{1} \cdots *_{n}}$ для всех $1 \leqslant k_{j} \leqslant m_{j}, j=1,2, \ldots, n$, можно утверждать, что найдутся, по крайней мере, $m_{1} \cdot m_{2} \ldots m_{n}$ членов последовательности $\left\{a_{\mathbf{k}}\right\}_{\mathbf{k} \in \mathbb{N} n}$, которые по модулю не меньше числа $a_{m_{1} \ldots m_{n}}^{*_{1} \ldots m_{n}}$ и, следовательно, $\beta_{m_{1}, \ldots, m_{n}}>$ $m_{1} \cdot m_{2} \ldots m_{n}$. Поэтому при $2<p$ имеем

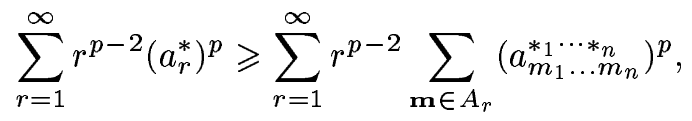

а при $p<2$

$$
\sum_{r=1}^{\infty} r^{p-2}\left(a_{r}^{*}\right)^{p} \leqslant \sum_{r=1}^{\infty} r^{p-2} \sum_{\mathbf{m} \in A_{r}}\left(a_{m_{1} \ldots m_{n}}^{*{ }_{1} \cdots m_{n}}\right)^{p}
$$

Принимая во внимание (11), получим, что из неравенств (9), (10) следуют соответственно (3), (4).

Следуюший пример показывает, что неравенства (9) и (3) не эквивалентны. 
ПримеР 2. Пусть $p>3,0<\varepsilon<p-3, p^{\prime}=p /(p-1)$,

$$
a_{k_{1} k_{2}}=\left(k_{1} k_{2}\right)^{-1 / p^{\prime}}\left(\ln \left(k_{1} k_{2}+1\right)\right)^{-(2+\varepsilon) / p}, \quad k_{1}, k_{2} \in \mathbb{N} .
$$

Очевидно, что

$$
\sum_{k_{1}=1}^{\infty} \sum_{k_{2}=1}^{\infty} k_{1}^{p-2} k_{2}^{p-2}\left(a_{k_{1} k_{2}}^{*_{1} *_{2}}\right)^{p}<\infty
$$

Пусть $A_{r}=\left\{\mathbf{m} \in \mathbb{N}^{2}: m_{1} m_{2}=r\right\}, r \in \mathbb{N}$. Как и ранее,

$$
\sum_{r=1}^{\infty} r^{p-2}\left(a_{r}^{*}\right)^{p}=\sum_{r=1}^{\infty} \sum_{\mathbf{m} \in A_{r}} \beta_{m_{1} m_{2}}^{p-2}\left(a_{m_{1}, m_{2}}^{*_{1} *_{2}}\right)^{p}
$$

Так как $\left(a_{k_{1} k_{2}}^{*_{1} *_{2}}\right)>\left(a_{m_{1} m_{2}}^{*_{1} *_{2}}\right)$ при $k_{1} \cdot k_{2}<m_{1} \cdot m_{2}$, то $\beta_{m_{1} m_{2}}>\sum_{s=1}^{r-1}\left|A_{s}\right|$ при $m_{1}$. $m_{2}>r$; следовательно,

$$
\sum_{r=1}^{\infty} r^{p-2}\left(a_{r}^{*}\right)^{p} \geqslant \sum_{r=1}^{\infty}\left(\sum_{s=1}^{r-1}\left|A_{s}\right|\right)^{p-2}\left|A_{r}\right| \frac{1}{r^{p-1}(\ln r+1)^{2+\varepsilon}} .
$$

Количество элементов $\left|A_{r}\right|$ во множестве $A_{r}$ не меньше количества различных делителей числа $r$. Известно, что сумма всех различных делителей чисел от 1 до $r$ асимптотически равна $r \ln r$. Поэтому

$$
\sum_{r=1}^{\infty} r^{p-2}\left(a_{r}^{*}\right)^{p} \geqslant c \sum_{r=1}^{\infty} \frac{\left|A_{r}\right|}{r(\ln r+1)^{4-p+\varepsilon}} \geqslant c \sum_{r=1}^{\infty} \frac{1}{r(\ln r+1)^{4-p+\varepsilon}}=\infty .
$$

Покажем теперь, что из неравенства (9) следует (5).

Пусть $2<p<\infty$. Заметим, что для любого $h: 1 \leqslant h \leqslant \infty$

$$
\begin{aligned}
\sum_{k_{1}=1}^{\infty} \cdots & \sum_{k_{n}=1}^{\infty} k_{1}^{p-2} \ldots k_{n}^{p-2}\left(a_{k_{1} \ldots k_{n}}^{*_{1} \cdots *_{n}}\right)^{p} \\
& \sim \sum_{k_{1}=0}^{\infty} \cdots \sum_{k_{n}=0}^{\infty} 2^{\left(\frac{1}{p^{\prime}}-\frac{1}{h}\right) p\left(k_{1}+\cdots+k_{n}\right)}\left(\sum_{m_{1}=2^{k_{1}}}^{2^{k_{1}+1}-1} \cdots \sum_{m_{n}=2^{k_{n}}}^{2^{k_{n}+1}-1}\left(a_{m_{1} \ldots m_{n}}^{*_{1} \cdots *_{n}}\right)^{h}\right)^{p / h} .
\end{aligned}
$$

Взяв $h=2$, получим

$$
\begin{aligned}
& \sum_{k_{1}=1}^{\infty} \cdots \sum_{k_{n}=1}^{\infty} k_{1}^{p-2} \ldots k_{n}^{p-2}\left(a_{k_{1} \ldots k_{n}}^{*_{1} \ldots *_{n}}\right)^{p} \\
& \sim \sum_{k_{1}=0}^{\infty} \cdots \sum_{k_{n}=0}^{\infty} 2^{\left(\frac{1}{p^{\prime}}-\frac{1}{2}\right) p\left(k_{1}+\cdots+k_{n}\right)}\left(\sum_{m_{1}=2^{k_{1}}}^{2^{k_{1}+1}-1} \cdots \sum_{m_{n}=2^{k_{n}}}^{2^{k_{n}+1}-1}\left(a_{m_{1} \ldots m_{n}}^{*_{1} \cdots m_{n}}\right)^{2}\right)^{p / 2} \\
& \leqslant \sum_{k_{1}=0}^{\infty} \cdots \sum_{k_{n}=0}^{\infty} 2^{\left(\frac{1}{2}-\frac{1}{p}\right) p\left(k_{1}+\cdots+k_{n}\right)}\left(\sum_{m_{1}=2^{k_{1}}}^{\infty} \cdots \sum_{m_{n}=2^{k_{n}}}^{\infty}\left(a_{m_{1} \cdots m_{n}}^{*_{1} \cdots *_{n}}\right)^{2}\right)^{p / 2} \\
& \leqslant \sum_{k_{1}=0}^{\infty} \cdots \sum_{k_{n}=0}^{\infty} 2^{\left(\frac{p}{2}-1\right)\left(k_{1}+\cdots+k_{n}\right)}\left(\sum_{m_{1}=2^{k_{1}}}^{\infty} \cdots \sum_{m_{n}=2^{k_{n}}}^{\infty}\left|a_{m_{1} \ldots m_{n}}\right|^{2}\right)^{p / 2} \\
& =\sum_{k_{1}=0}^{\infty} \cdots \sum_{k_{n}=0}^{\infty} 2^{\left(\frac{p}{2}-1\right)\left(k_{1}+\cdots+k_{n}\right)}\left(\sum_{\nu_{1}=k_{1}}^{\infty} \cdots \sum_{\nu_{n}=k_{n}}^{\infty} \sum_{m_{1}=2^{\nu_{1}}}^{2^{\nu_{1}+1}-1} \cdots \sum_{m_{n}=2^{\nu_{n}}}^{2^{\nu_{n}+1}-1}\left|a_{m_{1} \ldots m_{n}}\right|^{2}\right)^{p / 2} \text {. }
\end{aligned}
$$


Далее, применяя одно из обобщений неравенства Харди (см. [17]), последнее выражение оцениваем через

$$
\sum_{k_{1}=0}^{\infty} \cdots \sum_{k_{n}=0}^{\infty} 2^{\left(\frac{p}{2}-1\right)\left(k_{1}+\cdots+k_{n}\right)}\left(\sum_{m_{1}=2^{k_{1}}}^{2^{k_{1}+1}-1} \cdots \sum_{m_{n}=2^{k_{n}}}^{2^{k_{n}+1}-1}\left|a_{m_{1} \ldots m_{n}}\right|^{2}\right)^{p / 2}
$$

что и требовалось.

Я. С. Бугров в работе [18] для тригонометрических рядов получил неравенство: при $1<p_{n} \leqslant p_{n-1} \leqslant \cdots \leqslant p_{1}<2, f \sim \sum_{m \in \mathbb{Z}^{n}} a_{m} \exp \left(2 \pi i \sum_{r=1}^{n} m_{r} x_{r}\right), f \in L_{\mathbf{p}}[0,1]^{n}$ верно

$$
\begin{gathered}
\left(\sum_{m_{1} \in \mathbb{Z}}\left(\left|m_{1}\right|+1\right)^{p_{1}-2}\left(\ldots\left(\sum_{m_{n} \in \mathbb{N}}\left(\left|m_{n}\right|+1\right)^{p_{n}-2}\left|a_{m_{1} \ldots m_{n}}\right|^{p_{n}}\right)^{p_{n-1} / p_{n}} \ldots\right)^{p_{1} / p_{2}}\right)^{1 / p_{1}} \\
\leqslant c\left(\int_{0}^{1}\left(\ldots\left(\int_{0}^{1}\left|f\left(x_{1}, \ldots, x_{n}\right)\right|^{p_{1}} d x_{1}\right)^{p_{2} / p_{1}} \ldots\right)^{p_{n} / p_{n-1}} d x_{n}\right)^{1 / p_{n}} \cdot
\end{gathered}
$$

Покажем, что из теоремы 4 следует (12). Не уменьшая общности, можно считать, что $n=2$. Принимая во внимание, что $p_{j}<2$, а следовательно, последовательности $\left\{\left(\left|m_{j}\right|+1\right)^{p_{j}-2}\right\}, j=1,2$, симметрично убывающие, получим

$$
\begin{aligned}
& I=\left(\sum_{m_{1} \in \mathbb{Z}}\left(\left|m_{1}\right|+1\right)^{p_{1}-2}\left(\left(\sum_{m_{2} \in \mathbb{Z}}\left(\left|m_{2}\right|+1\right)^{p_{2}-2}\left|a_{m_{1} m_{2}}\right|^{p_{2}}\right)^{\frac{1}{p_{2}}}\right)^{p_{1}}\right)^{\frac{1}{p_{1}}} \\
& \leqslant 4\left(\sum_{k_{1}=1}^{\infty} k_{1}^{p_{1}-2}\left(\left[\left(\sum_{k_{2}=1}^{\infty} k_{2}^{p_{2}-2}\left|a_{m_{1} k_{2}}^{*_{2}}\right|^{p_{2}}\right)^{\frac{1}{p_{2}}}\right]_{k_{1}}^{*_{1}}\right)^{p_{1}}\right)^{\frac{1}{p_{1}}} \\
& \leqslant 4\left(\sum_{k_{1}=1}^{\infty} k_{1}^{p_{1}-2} \sup _{\substack{|e|=k_{1} \\
e \subset \mathbb{Z}}}\left(\frac{1}{|e|} \sum_{m_{1} \in e}\left(\sum_{k_{2}=1}^{\infty} k_{2}^{p_{2}-2}\left|a_{m_{1} k_{2}}^{*_{2}}\right|^{p_{2}}\right)^{\frac{2}{p_{2}}}\right)^{\frac{p_{1}}{2}}\right)^{\frac{1}{p_{1}}} \\
& \leqslant 4\left(\sum_{k_{1}=1}^{\infty} k_{1}^{p_{1}-2}\left(\sum_{k_{2}=1}^{\infty} k_{2}^{p_{2}-2} \sup _{\substack{|e|=k_{1} \\
e \subset \mathbb{Z}}}\left(\frac{1}{|e|} \sum_{m_{1} \in e}\left|a_{m_{1} k_{2}}^{*_{2}}\right|^{2}\right)^{\frac{p_{2}}{2}}\right)^{\frac{p_{1}}{p_{2}}}\right)^{\frac{1}{p_{1}}} \\
& =4\left(\sum_{k_{1}=1}^{\infty} k_{1}^{p_{1}-2}\left(\sum_{k_{2}=1}^{\infty} k_{2}^{p_{2}-2}\left(\frac{1}{k_{1}} \sum_{r_{1}=1}^{k_{1}}\left(a_{r_{1} k_{2}}^{*_{2} *_{1}}\right)^{2}\right)^{\frac{p_{2}}{2}}\right)^{\frac{p_{1}}{p_{2}}}\right)^{\frac{1}{p_{1}}} \text {. }
\end{aligned}
$$

Так как $\left(\frac{1}{k} \sum_{r=1}^{k}\left(b_{r}^{*}\right)^{2}\right)^{\frac{1}{2}} \leqslant c \frac{1}{k^{\frac{1}{2}}} \sum_{r=1}^{k} r^{-\frac{1}{2}} b_{r}^{*}$, где $c$ не зависит от $k$ и последовательности $\left\{b_{r}\right\}$, то

$$
\begin{aligned}
I & \leqslant c_{1}\left(\sum_{k_{1}=1}^{\infty} k_{1}^{p_{1}-2}\left(\sum_{k_{2}=1}^{\infty} k_{2}^{p_{2}-2}\left(\frac{1}{k_{1}^{\frac{1}{2}}} \sum_{r=1}^{k_{1}} r_{1}^{-\frac{1}{2}} a_{r_{1} k_{2}}^{*_{2} *_{1}}\right)^{p_{2}}\right)^{\frac{p_{1}}{p_{2}}}\right)^{\frac{1}{p_{1}}} \\
& \leqslant c_{1}\left(\sum_{k_{1}=1}^{\infty} k_{1}^{p_{1}-2}\left(\frac{1}{k_{1}^{\frac{1}{2}}} \sum_{r_{1}=1}^{k_{1}} r_{1}^{-\frac{1}{2}}\left(\sum_{k_{2}=1}^{\infty} k_{2}^{p_{2}-2}\left(a_{r_{1} k_{2}}^{*_{2} *_{1}}\right)^{p_{2}}\right)^{\frac{1}{p_{2}}}\right)^{p_{1}}\right)^{\frac{1}{p_{1}}} \\
& \leqslant c_{2}\left(\sum_{k_{1}=1}^{\infty} k_{1}^{p_{1}-2}\left(\sum_{k_{2}=1}^{\infty} k_{2}^{p_{2}-2}\left(a_{k_{1} k_{2}}^{*_{2} *_{1}}\right)^{p_{2}}\right)^{\frac{p_{1}}{p_{2}}}\right)^{\frac{1}{p_{1}}} .
\end{aligned}
$$


Теперь воспользуемся теоремой 4:

$$
\begin{aligned}
I & \leqslant c_{3}\left(\int_{0}^{1}\left(\int_{0}^{1}\left(f^{*_{1}, *_{2}}\left(t_{1}, t_{2}\right)\right)^{p_{2}} d t_{2}\right)^{\frac{p_{1}}{p_{2}}} d t_{1}\right)^{\frac{1}{p_{1}}} \\
& =c_{3}\left(\int_{0}^{1}\left(\int_{0}^{1}\left(f^{*_{1}}\left(t_{1}, x_{2}\right)\right)^{p_{2}} d x_{2}\right)^{\frac{p_{1}}{p_{2}}} d t_{1}\right)^{\frac{1}{p_{1}}} \\
& \leqslant c_{3}\left(\int_{0}^{1}\left(\int_{0}^{1}\left|f\left(x_{1}, x_{2}\right)\right|^{p_{1}} d x_{1}\right)^{\frac{p_{2}}{p_{1}}} d x_{2}\right)^{\frac{1}{p_{2}}} .
\end{aligned}
$$

\section{СПИСОК ЦИТИРОВАННОЙ ЛИТЕРАТУРЫ}

[1] Yoshikawa F. Remarks on the theory of interpolation spaces // J. Fac. Sci. Univ. Tokyo. 1968. Sec. I. V. 15. P. 209-251.

[2] Sparr G. Interpolation of several Banach spaces // Annali Mat. Pura Appl. 1974. V. 99. P. 247-316.

[3] Fernandez D. L. Lorentz spaces, with mixed norms // J. Funct. Anal. 1977. V. 25. № 2. P. 128-146.

[4] Fernandez D. L. Interpolation of $2^{n}$ Banach spaces // Studia Math. (PRL). 1979. V. 65. № 2. P. 175-201.

[5] Cobus F., Peetre J. Interpolation compact operators: The multidimensional case // Proc. London Math. Soc. 1991. V. 63. № 2. P. 371-400.

[6] Крепкогорский В. Л. Контрпримеры к теории операторов в пространствах со смешанной нормой // Деп. ВИНИТИ № 2963-80. Казань, 1980.

[7] Blozinski A. P. Multivariate rearrangements and Banach function spaces with mixed norms // Trans. Amer. Math. Soc. 1981. V. 263. №1. P. 149-167.

[8] Asekritova I., Krugljak N. On equivalence of K-end J-methods for $(n+1)$-tuples of Banach spaces // Stud. Math. 1997. V. 122. № 2. P. 99-116.

[9] Nursultanov E. D. Interpolation properties of some anisotropic spaces and Hardy-Littlewood type inequalities // East J. Approx. 1998. V. 4. № 2. P. 243-275.

[10] Нурсултанов Е. Д. О коэффициентах кратных рядов Фурье из $L_{p}$ пространств // Изв. PAH. 2000. T. 64. №1. C. 95-122.

[11] Hörmander L. Estimates for translation-invariant operators in $L_{p}$ spaces // Acta. Math. 1960. V. 104. P. 93-140.

[12] Нурсултанов Е. Д. О нижней оценке мультипликаторов Фурье из $M_{p}^{q} / /$ Матем. заметки. 1997. Т. 62. №6. С. 945-950.

[13] Зигмунд А. Тригонометрические ряды. Т. 2. М.: Мир, 1965.

[14] Pitt H. R. Theorems on Fourier series and Lower series // Duke Math. J. 1937. №3. P. $747-755$.

[15] Темляков В. Н. Приближение функции с ограниченной смешанной производной // Тр. МИАН СССР. 1986. Т. 178. С. 1-112.

[16] Смаилов Е. С. Мультипликаторы Фурье, теоремы вложения и смежные с ними вопросы. Дисс. ... д. ф.-м.н.. Алматы, 1997.

[17] Потапов М. К. Теоремы Харди-Литтлвуда, Марцинкевича, Литтлвуда-Пэли, приближение углом и вложение некоторых классов функций // Matematika. 1972. T. 14. № 2. С. 339-362.

[18] Бугров Я. С. Абсолютная сходимость преобразования Фурье и абсолютная сходимость кратных рядов Фурье // Докл. АН СССР. 1988. Т. 302. № 5. С. 1033-1035.

Казахстанский филиал МГУ им. М. В. Ломоносова, 